\title{
O AUMENTO DA LUCRATIVIDADE EXPANDE A ACUMULAÇÃO DE CAPITAL? UMA ANÁLISE DE CAUSALIDADE DE GRANGER PARA PAÍSES DA OCDE*
}

\author{
Adalmir Marquetti ${ }^{* *}$
}

\section{Daniel Koshiyama ${ }^{* * *}$}

\section{Denilson Alencastro ${ }^{* * *}$}

\begin{abstract}
RESUMO O objetivo deste trabalho é testar a hipótese clássico-marxiana de ligação causal entre a taxa de lucro e a taxa de acumulação de capital para um conjunto de 20 países da OCDE. A metodologia utilizada baseia-se no procedimento proposto por Toda e Yamamoto (1995) para testar a hipótese de não causalidade de Granger. A especificação de teste empregada, derivada a partir da equação de Cambridge, envolve três variáveis: a taxa de lucro, a taxa de acumulação e a taxa de investimento. A consideração da variável investimento permite comparações entre as tradições clássico-marxiana e pós-keynesianas. Os resultados para a Austrália, a Dinamarca, os EUA, a Finlândia e a Irlanda são consistentes com a concepção clássico-marxiana. Por outro lado, os resultados para o Canadá, a Coreia do Sul, a Grécia e a Suécia são parcialmente consistentes com a tradição pós-keynesiana.
\end{abstract}

Palavras-chave: taxa de lucro; taxa de acumulação; causalidade de Granger

Código JEL: E11; E12

\footnotetext{
* Artigo recebido em 5 de setembro de 2007 e aprovado em 11 de setembro de 2009. Os autores agradecem os comentários dos pareceristas anônimos da Revista, os quais muito contribuíram para melhorar a versão original do texto. Os erros remanescentes são de inteira responsabilidade dos autores.

** Doutor em Economia, Professor Titular da PUCRS, e-mail: aam@pucrs.br

*** Mestre em Economia do Desenvolvimento (PPGE/PUCRS), e-mail: daniel@sinon.com.br

**** Mestrando do PPGE/PUCRS, e-mail: denilson@geralasset.com.br
} 


\title{
DOES INCREASING PROFITABILITY RISE CAPITAL ACCUMULATION? A GRANGER CAUSALITY ANALYSIS ON OECD COUNTRIES
}

\begin{abstract}
The purpose of this paper is to test the classical-Marxian hypothesis of causal linkages between profit rate and the accumulation of capital for a dataset of 20 OECD countries. The procedure proposed by Toda and Yamamoto (1995) to test for the Granger non-causality hypotheses is employed in the statistical procedure. The test specification, derived from the Cambridge equation, involve three variables: profit rate, accumulation of capital and investment rate. The consideration of the investment rate allows a comparison between the classical-Marxian and the post-Keynesian traditions. For the cases of Australia, Denmark, USA, Finland and Ireland, the results provide empirical support for the classical-Marxian conception. On the other hand, in the cases of Canada, South Korea, Greece and Sweden, the results support the post-Keynesian tradition.
\end{abstract}

Key words: profit rate; accumulation rate; Granger causality 


\section{INTRODUÇÃO}

Os economistas heterodoxos têm diferentes concepções acerca da relação causal entre lucratividade, investimento e acumulação de capital. A abordagem clássico-marxiana argumenta que a principal força que impulsiona a acumulação de capital é a lucratividade. Nessa tradição, a evolução da taxa de lucro determina a trajetória da acumulação de capital, dada a propensão a poupar dos capitalistas. A abordagem pós-keynesiana, por sua vez, enxerga uma relação de dependência da lucratividade e da acumulação de capital em relação ao investimento.

O objetivo deste artigo é testar a hipótese clássico-marxiana de ligação causal entre taxa de lucro e taxa de acumulação para 20 países da OCDE entre 1963 e 2004. Para três países o ano final da análise é 2003. O teste de não causalidade de Granger (1969) é bem adequado para se estudar a ideia de que mudanças na lucratividade antecipam os movimentos da acumulação de capital. O conceito de causalidade de Granger é definido em termos da previsibilidade das séries temporais; a causalidade é testada no sentido de qual variável ajuda a prever a outra.

A análise empírica é realizada a partir de uma versão linear da equação de Cambridge na qual a taxa de poupança é substituída pela taxa de investimento. $\mathrm{O}$ teste de não causalidade de Granger envolve três variáveis: a taxa de lucro, a taxa de acumulação e a taxa de investimento. A omissão de uma variável relevante tal como a taxa de lucro pode causar um viés de especificação no modelo. Além disso, a consideração dessa variável permite comparações entre as tradições clássico-marxiana e pós-keynesiana. Gordon (1994) e Lavoie, Rodríguez e Seccareccia (2004) comparam modelos macroeconômicos nas duas concepções.

Neste estudo, empregamos o procedimento proposto por Toda e Yamamoto (1995) para testar a hipótese de não causalidade de Granger. Trata-se de uma abordagem metodológica relativamente recente que leva em consideração a informação de longo prazo presente no sistema.

O artigo está organizado em seis seções, além da introdução. A segunda seção apresenta um modelo de crescimento simplificado inspirado na tradição clássico-marxiana. A terceira discute a especificação econométrica utilizada. A quarta seção aborda aspectos relacionados à base de dados. A seção cinco apresenta o método de teste de não causalidade de Granger proposto 
por Toda e Yamamoto (1995). A seção seis apresenta os resultados obtidos e sua interpretação. Por fim, na sétima seção são realizadas as considerações finais.

\section{UM MODELO SIMPLIFICADO DE CRESCIMENTO}

Esta seção apresenta um modelo de crescimento de longo prazo simplificado inspirado na tradição clássico-marxiana. A despeito de sua simplicidade, ele é capaz de mostrar a relação de causalidade básica entre as variáveis consideradas na análise. O modelo aqui considerado foi originalmente desenvolvido por Marglin (1986) e Foley e Michl (1999).

Considera-se que somente uma mercadoria é produzida. Para produzi-la são necessários capital, $K$, e trabalho, $N$. O produto, $X$, pode ser acumulado na forma de capital, $K$, o qual deprecia a taxa $d$, ou consumido. A produção leva tempo, os insumos são empregados no início do período, a mercadoria torna-se disponível no fim do período. A produtividade do trabalho, $x$, é definida pela razão $X / N$.

A economia em questão é fechada e sem governo e produz um excedente. A produção é distribuída na forma de renda. Os indivíduos pertencem ou à classe capitalista, cuja renda assume a forma de lucros, ou à classe trabalhadora, cuja renda assume a forma de salários. Existe livre concorrência e as firmas têm acesso à mesma tecnologia e, portanto, existe equalização das taxas de lucro e a economia utiliza plenamente sua capacidade produtiva. A competição no mercado de trabalho também garante a equalização dos salários. Partindo da definição do produto pela ótica da renda, o trade-off entre salários e lucros é expresso pela fronteira salário-lucro:

$$
w=x-(r+d) k
$$

onde $w$ representa o salário real; $k$, a razão capital-trabalho; $r$, a taxa de lucro líquida; e $d$, a taxa de depreciação. A taxa de lucro máxima, correspondente a $w=0$, é igual à razão produto-capital, $\rho=x / k$. O salário real máximo, correspondente a $r+d=0$, é igual a $x$.

O produto pode ser empregado tanto para o investimento quanto para o consumo. O crescimento do produto depende da acumulação de capital, a razão entre o investimento e o estoque de capital. Partindo da definição do 
produto pela ótica da renda, o trade-offentre o investimento e o consumo é expresso pela fronteira consumo-crescimento:

$$
c=x-\left(g_{k}+d\right) k
$$

onde $c$ denota o consumo social por trabalhador e $g_{k}$, a acumulação de capital. A taxa máxima de acumulação de capital, correspondente a $c=0$, é igual a $\rho$. O consumo máximo por trabalhador, correspondente a $g_{k}+d=0$, é igual a $x$.

As esferas da distribuição e do crescimento são ligadas pela hipótese acerca das decisões de poupança e investimento. Por uma questão de simplicidade, assume-se que os trabalhadores consomem todo o seu salário e que os capitalistas poupam uma proporção $s$ dos seus lucros, sendo $1>s>0$. A função de poupança pode ser escrita como:

$$
S=s(R+D)
$$

onde $S$ é a poupança total, $R$ representa os lucros líquidos e $D$ é a depreciação. Empregando a suposição de que o investimento é igual à poupança, obtém-se:

$$
I=s Z=s(R+D)
$$

onde $I$ denota o investimento. Dividindo os dois lados pelo estoque de capital, a equação de Cambridge pode ser escrita como:

$$
g_{k}+d=s(r+d)
$$

A equação de Cambridge informa que a acumulação do capital é função positiva da taxa de poupança e da taxa de lucro. As equações (1), (2) e (5) fornecem a estrutura básica para diferentes tradições econômicas. Estas têm fechamentos distintos para seus modelos (Marglin, 1986; Dutt, 1990). Para os limites teóricos do fechamento neoclássico, ver Gontijo (1998).

Na concepção clássico-marxiana, a distribuição é determinada exogenamente. A oferta de trabalho é considerada totalmente elástica no nível de 
salário de subsistência. Ricardo explica esse formato considerando a teoria da população de Malthus, enquanto Marx postula a existência de um exército industrial de reserva. Para Marx, o salário real é determinado por fatores históricos e institucionais, com a oferta de trabalho se adaptando às necessidades da acumulação de capital.

Seguindo essa tradição e em alinhamento com a experiência histórica, emprega-se a suposição de que a parcela de salário, $1-\pi$, é constante. O salário real é determinado por:

$$
w=(1-\pi) x
$$

Portanto, no modelo aqui apresentado, a esfera da distribuição é determinada exogenamente e a esfera do crescimento é determinada endogenamente. As variáveis endógenas no modelo são obtidas em passos sequenciais. Primeiramente, o salário real é determinado pela equação (6); então, a taxa de lucro é calculada pela equação (1); o próximo passo é a determinação da acumulação de capital através de (5); e, finalmente, o consumo social

Figura 1: Representação do modelo de crescimento clássico-marxiano

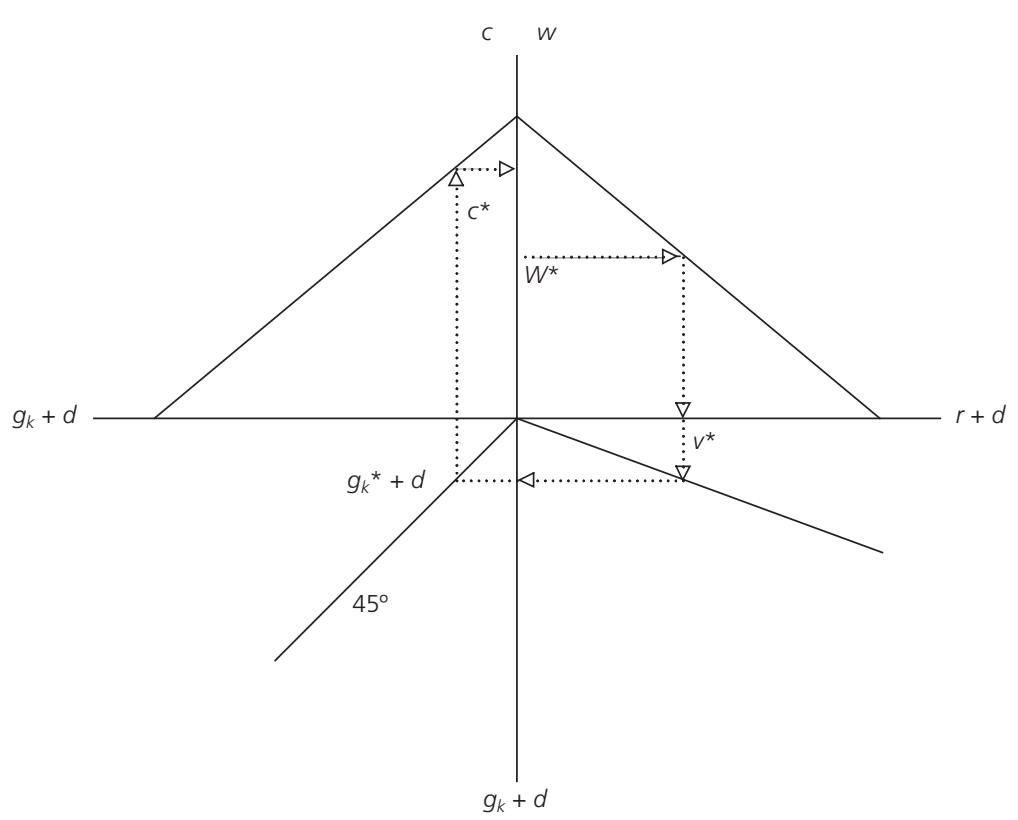


por trabalhador é computado por (2). A figura 1 oferece uma descrição diagramática do modelo.

Na concepção clássico-marxiana, a taxa de lucro é definida pela distribuição e pela tecnologia. A acumulação de capital depende positivamente da lucratividade e da taxa de poupança. Movimentos na taxa de lucro antecipam as mudanças na acumulação de capital.

\section{ESPECIFICAÇÃO ECONOMÉTRICA}

O ponto de partida para a análise econométrica é a equação de Cambridge. Essa equação pode ser expressa em termos logarítmicos como:

$\ln (g k+d)=\ln (s)+\ln (v)$

onde $v=r+d$.

Essa expressão, no entanto, possui um problema. As séries da taxa normal de acumulação e da taxa normal de lucro não estão disponíveis e, portanto, variáveis substitutas precisam ser encontradas. Inicialmente, assume-se que a taxa observada de poupança corresponde à taxa normal de poupança. A falta de séries diretamente observáveis da taxa normal de acumulação pode ser resolvida caso seja possível substituí-las por variáveis observáveis. ${ }^{1} \mathrm{Na}$ concepção clássico-marxiana, os valores observados oscilam em torno dos valores normais. Dessa forma, é possível definir:

$\varepsilon=v / v_{a} \quad$ e $\quad \xi=g^{K+d} /\left(g^{K}+d\right)_{a}$

onde o subscrito a denota os valores observados. Aplicando logaritmos, podemos reescrever essas expressões como:

$\ln (v)=\ln \left(v_{a}\right)+\ln (\varepsilon) \quad$ e $\quad \ln \left(g_{K}+d\right)=\ln \left(g_{K}+d\right)_{a}+\ln (\xi)$

Uma das propriedades esperadas dos termos $\ln (\varepsilon)$ e $\ln (\xi)$ é a de que eles sejam estacionários. Portanto, as variáveis normais são substituídas por $\ln$ $\left(v_{a}\right)$ e $\ln (\varepsilon)$ e $\ln \left(g_{K}+d\right)_{a}+\ln (\xi)$ na equação $(8)$, que passa a ser:

$\ln \left(g_{K}+d\right)_{a}=\ln (s)+\ln \left(v_{a}\right)+\psi$

onde $\psi=\ln (\varepsilon)-\ln (\xi)$ e contém somente componentes estacionários. 
Uma próxima especificação para essa equação envolve uma hipótese acerca da taxa de poupança. Sob a suposição de que a taxa de poupança e a taxa de investimento são cointegradas, é possível considerar:

$\ln (s)=\ln (i)+\varphi$

onde $\varphi$ representa um termo de erro com as propriedades desejáveis e $i$ representa a taxa de investimento, definida como a razão entre o investimento e o produto. Empregando (11), é possível reescrever (10) como:

$\ln \left(g_{K}+d\right)_{a}=\ln (i)+\ln \left(v_{a}\right)+\tau$

onde $\tau=\varphi+\psi$ e contém apenas componentes estacionários.

Em resumo, existem duas variantes para o teste de não causalidade de Granger entre a taxa de lucro e a taxa de acumulação, quais sejam: a especificação (10), que envolve a taxa de poupança, e a especificação (13), que é uma extensão daquela e considera a taxa de investimento.

A especificação (12) é preferida tendo em vista a importância da variável investimento na tradição pós-keynesiana. Nessa concepção, o investimento é a "variável crucial no sistema" (Kregel, 1972, p. 49). Portanto, a especificação (12) permite uma comparação entre as tradições clássico-marxiana e pós-keynesiana.

\section{OS DADOS}

A base de dados contém observações anuais para o período entre 1963 e 2004 para as taxas de lucro, a acumulação de capital e a taxa de investimento para um conjunto de 20 países membros da OCDE, quais sejam: Austrália, Áustria, Canadá, Coreia do Sul, Dinamarca, EUA, Finlândia, França, Grécia, Holanda, Irlanda, Islândia, Itália, Japão, Luxemburgo, Noruega, Nova Zelândia, Reino Unido, Suécia e Suíça. Para três países, o último ano da análise é 2003. A fonte de informações é a Extended Penn World Table 2.2, um banco de dados compilado a partir de Summers, Heston e Aten (2006) e outras bases de dados.

A taxa de lucro é medida como a razão entre os lucros brutos e o estoque líquido de capital fixo. A taxa de investimento é a razão entre investi- 
mento bruto e produto interno bruto. Por sua vez, a acumulação do capital é medida como a razão entre o investimento bruto e o estoque líquido de capital fixo.

\section{O MÉTODO DE TESTE}

O teste de não causalidade de Granger (1969) é um método adequado para se estudar a ideia de que mudanças na lucratividade antecipam os movimentos da acumulação de capital. O conceito de causalidade de Granger diz respeito à capacidade de uma variável em auxiliar na previsão do comportamento de uma outra variável de interesse. Trata-se da existência de uma precedência temporal estatisticamente significante na explicação de uma dada variável.

No entanto, quando as variáveis de interesse são integradas, os métodos tradicionais para o teste de não causalidade de Granger (1969) não são válidos, pois as estatísticas de teste não seguem uma distribuição padrão (Rambaldi e Doran, 1996, p. 3). Sendo assim, a maioria dos estudos aplicados recentes adota a seguinte estratégia de teste:

(1) Aplicam-se os testes de raiz unitária;

(2) Se as séries forem estacionárias, o teste de não causalidade é realizado no contexto de um modelo de vetor autorregressivo (VAR) em níveis;

(3) Se as séries forem não estacionárias, aplica-se um teste de cointegração;

(4) Se não houver cointegração, o teste de não causalidade é realizado no contexto de um modelo VAR em primeiras diferenças;

(5) Se houver cointegração, o teste de não causalidade é realizado no contexto de um modelo de correção de erro vetorial (VECM);

(6) Adicionalmente, alguns autores utilizam funções de impulso-resposta e/ou métodos de decomposição da variância para esclarecer melhor o impacto de choques sobre as variáveis endógenas.

Contudo, a abordagem discutida apresenta dois inconvenientes. Primeiro, a implementação do VECM depende de pré-testes de raiz unitária e cointegração. E, conforme discutido na literatura especializada, os prétestes possuem baixo poder, o que gera maior grau de incerteza com rela- 
ção à confiabilidade dos resultados dos testes de não causalidade. Ademais, os testes de cointegração admitem distintas especificações, e muitas vezes elas produzem resultados que são conflitantes entre si, ensejando certo grau de arbitrariedade. Segundo, nos casos em que não há cointegração, é realizada a diferenciação das séries, o que resulta na perda de informação de longo prazo. ${ }^{2}$

No entanto, Toda e Yamamoto (1995) desenvolveram um teste alternativo capaz de superar essas deficiências. Esse teste pode ser utilizado em sistemas possivelmente integrados ou cointegrados, sem a necessidade de prétestes de raiz unitária ou cointegração, ${ }^{3}$ e também permite que as variáveis possuam ordens de integração distintas. Além disso, Yamada e Toda (1998) verificaram que o teste é o mais adequado quando se está trabalhando com amostras relativamente pequenas. Sendo assim, essa é a abordagem metodológica empregada no artigo.

O procedimento de Toda e Yamamoto (1995) consiste em um teste de Wald modificado (MWALD), no qual são testadas restrições nos parâmetros de um modelo VAR. O teste é realizado diretamente nos estimadores de mínimos quadrados de um VAR aumentado em níveis. Toda e Yamamoto (1995) demonstraram que, em sistemas integrados ou cointegrados, o teste Wald para restrições lineares nos parâmetros de um VAR $(z)$ tem distribuição assimptótica chi-quadrado $\left(x^{2}\right)$, independentemente da não estacionaridade envolvida no sistema, quando um $\operatorname{VAR}\left(z+e_{\text {max }}\right)$ é estimado, onde $e_{\text {max }}$ é a ordem máxima de integração das séries. O teste de restrições de Wald para a hipótese de não causalidade de Granger é realizado nos $z$ primeiros coeficientes, sendo os últimos $e_{\text {max }}$ coeficientes não considerados. Essas defasagens extras, no entanto, são necessárias para assegurar que a estatística de teste possua distribuição assimptótica chi-quadrado $\left(x^{2}\right)$.

Portanto, o teste de não causalidade de Granger sugerido por Toda e Yamamoto (1995) envolve três passos. O primeiro é a definição do número ótimo de defasagens $(z)$ e da ordem máxima de integração do sistema $\left(e_{\max }\right)$. O número ótimo de defasagens pode ser selecionado por meio do Critério de Informação de Schwarz (SIC) ou do Critério de Informação de Akaike (AIC), dentre outros. ${ }^{4}$ Quanto à ordem máxima de integração do sistema, 
esta pode ser definida de duas maneiras: (a) alguns autores, a fim de evitar possíveis vieses decorrentes dos pré-testes, simplesmente definem $e_{\max }=1$ com base no pressuposto de que as variáveis macroeconômicas são geralmente I (1); (b) outros autores examinam as propriedades univariadas das séries temporais empregando o teste de Dickey-Fuller aumentado (ADF) e/ ou o teste de Phillips-Perron (PP), dentre outros métodos.

O segundo passo é a estimação de um VAR em níveis com um total de $\left(z+e_{\max }\right)$ defasagens, conforme a seguir:

$$
\begin{aligned}
& \left(g_{k}+d\right)_{a}=c_{1}+\alpha_{1 j} \sum_{j=1}^{z}\left(g_{k}+d\right)_{a j}+\delta_{1 j} \sum_{j=1}^{z} v_{a j}+\beta_{1 j} \sum_{j=1}^{z} i_{a j}+\alpha_{1 l} \sum_{l=z+1}^{e}\left(g_{k}+d\right)_{a}+\delta_{1 l} \\
& \sum_{l=z+1}^{e} v_{a}+\beta_{1 l} \sum_{l=z+1}^{e} i_{a}+\tau_{1 t} \\
& v_{a}=c_{2}+\alpha_{2 j} \sum_{j=1}^{z}\left(g_{k}+d\right)_{a j}+\delta_{2 j} \sum_{j=1}^{z} v_{a j}+\beta_{2 j} \sum_{j=1}^{z} i_{a j}+\alpha{ }_{2 l} \sum_{l=z+1}^{e}\left(g_{k}+d\right)_{a}+\delta_{2 l} \\
& \sum_{l=z+1}^{e} v_{\mathrm{a}}+\beta_{2 l} \sum_{l=z+1}^{e} i_{a}+\tau_{2 t} \\
& i_{a}=c_{3}+\alpha_{3 j} \sum_{j=1}^{z}\left(g_{k}+d\right)_{a j}+\delta_{3 j} \sum_{j=1}^{z} v_{a j}+\beta_{3 j} \sum_{j=1}^{z} i_{a j}+\alpha_{3 l} \sum_{l=z+1}^{e}\left(g_{k}+d\right)_{a}+\delta_{3 l} \\
& \sum_{l=z+1}^{e} v_{a}+\beta_{3 l} \sum_{l=z+1}^{e} i_{a}+\tau_{3 t}
\end{aligned}
$$

O terceiro passo é a aplicação do teste de restrições de Wald nos $z$ primeiros coeficientes para testar a hipótese de não causalidade de Granger. Conclui-se que a taxa de acumulação "Granger-causa" a taxa de lucro e a taxa de investimento se, respectivamente, as hipóteses $H_{0}: \alpha_{2 j}=0$ e $H_{0}: \alpha_{3 j}=0$ são rejeitadas. A taxa de lucro Granger-causa a taxa de acumulação e a taxa de investimento se, respectivamente as hipóteses $H_{0}: \delta_{1 j}=0$ e $H_{0}: \delta_{3 j}=0$ são rejeitadas. Similarmente, a taxa de investimento Granger-causa a taxa de acumulação e a taxa de lucro se, respectivamente, as hipóteses $H_{0}: \beta_{1 j}=0$ e $H_{0}: \beta_{2 j}=0$ são rejeitadas. 


\section{RESULTADOS E DISCUSSÃO}

Para ilustrar o procedimento do teste, utiliza-se o exemplo dos EUA. Por meio da aplicação do teste Dickey-Fuller aumentado, ${ }^{5}$ é rejeitada a hipótese nula de duas raízes unitárias para as séries das taxas de lucro e de investimento. Contudo, não é rejeitada a hipótese nula de uma raiz unitária, concluindo que as séries são integradas de ordem um, I (1). No que se refere à taxa de acumulação de capital, são rejeitadas as hipóteses nulas de duas raízes unitárias, bem como a hipótese nula de uma raiz unitária, concluindo que a série é I (0). Sendo assim, a ordem máxima de integração do sistema $\left(e_{\max }\right)$ é um. Quanto ao número ótimo de defasagens, o Critério de Informação de Schwarz indicou a utilização de apenas um período (i.e., $z=1$ ). Dessa forma, a análise é empreendida utilizando-se um VAR $(1+1=2)$.

As propriedades univariadas das séries temporais para todos os países analisados, examinadas por meio do teste VAR, são apresentadas na tabela A1 do Anexo. ${ }^{6} \mathrm{Na}$ tabela A2 são reportadas a ordem máxima de integração do sistema $\left(e_{\max }\right)$ e o número ótimo de defasagens $(z)$ para os países da amostra. Observa-se que $z=1$ e $e_{\max }=1$ em todos os casos estudados, indicando a utilização de um VAR (2) para a realização dos testes de não causalidade.

No entanto, também são apresentados os resultados obtidos para um VAR (3), em virtude dos seguintes aspectos:

(a) o teste de não causalidade de Granger é sensível ao número de defasagens empregadas; ${ }^{7}$

(b) embora o SIC indique a estrutura de defasagens ótima para o sistema VAR como um todo, essa estrutura pode não ser a melhor para cada equação individualmente;

(c) a inclusão de defasagens adicionais pode eliminar eventuais problemas identificados no diagnóstico dos resíduos.

A propósito, cabe mencionar que, para verificar se o erro estimado tem as características necessárias a uma estimação robusta e assegurar a confiabilidade dos resultados, foram realizados os testes de diagnóstico padrão, quais sejam: o teste LM de autocorrelação residual (de primeira e segunda ordem), o teste de White de heterocedasticidade e o teste de normalidade dos resíduos. ${ }^{8}$ Dos 20 países analisados: nove países não apresentaram qualquer problema; cinco países apresentaram problemas somente em uma das duas especificações dos testes empregados; seis países (Coreia do Sul, Ho- 
landa, Itália, Noruega, Reino Unido e Suíça) apresentaram problemas nas duas especificações.

Os resultados completos dos testes de não causalidade são reportados nas tabelas A3, A4 e A5 do Anexo. Eventualmente, os resultados obtidos por meio de uma e outra especificação de teste - VAR (2) e VAR (3) - poderão ser contraditórios. Nesse caso, adotou-se a prática convencional de dar preferência à rejeição da hipótese nula, em detrimento da não rejeição.

$\mathrm{Na}$ situação em que se detectou a violação das propriedades desejáveis dos resíduos, mas apenas em uma das especificações utilizadas, foram desconsiderados os resultados gerados pela especificação com problema, assegurando-se a confiabilidade dos testes.

Já nos seis casos que apresentaram problemas no diagnóstico dos resíduos nas duas especificações (identificados no quadro 1), optou-se por reportar os resultados ao invés de simplesmente descartá-los. No entanto, eles são meramente sugestivos e devem ser interpretados com cautela, sendo necessário realizar novas investigações para confirmar ou refutar os resultados obtidos nesses seis casos.

Retomando o exemplo dos EUA, os resultados sugerem que: (a) a taxa de acumulação não Granger-causa a taxa de lucro e a taxa de investimento; (b) a taxa de investimento não Granger-causa a taxa de acumulação e a taxa de lucro e; (c) a taxa de lucro Granger-causa a taxa de acumulação e a taxa de investimento. Portanto, conclui-se que há uma causalidade de Granger unidirecional da taxa de lucro para a taxa de acumulação e para a taxa de investimento na economia norte-americana no período 1963-2004. Os resultados são consistentes com a concepção clássico-marxiana, para a qual a lucratividade é a principal força propulsora da acumulação de capital.

O quadro 1, elaborado a partir dos testes reportados no Anexo, apresenta um resumo das relações de causalidade de Granger identificadas nos países da amostra.

Os resultados para a Austrália, Dinamarca, Finlândia e Irlanda também corroboram a hipótese clássico-marxiana. Nesses casos, houve causalidade de Granger unidirecional da taxa de lucro para a taxa de investimento e para a acumulação de capital.

Por outro lado, em quatro casos encontraram-se resultados que são parcialmente consistentes com a tradição pós-keynesiana. Para o Canadá, verificou-se uma relação de causalidade unidirecional da taxa de investimento 
Quadro 1: Relações de causalidade de Granger

\begin{tabular}{|c|c|c|c|c|}
\hline País & Período & $\begin{array}{c}\text { Taxa de lucro } \\
\mathrm{X} \\
\text { Acumulação } \\
\text { de capital }\end{array}$ & $\begin{array}{c}\text { Taxa de lucro } \\
\mathrm{X} \\
\text { Taxa de } \\
\text { investimento }\end{array}$ & $\begin{array}{c}\text { Taxa de investimento } \\
X \\
\text { Acumulação } \\
\text { de capital }\end{array}$ \\
\hline Austrália & 1963-04 & $\ln \left(v_{a}\right) \rightarrow \ln \left(g_{K}+d\right)_{a}$ & $\ln \left(v_{a}\right) \rightarrow \ln \left(i_{a}\right)$ & - \\
\hline Dinamarca & 1963-04 & $\ln \left(v_{a}\right) \rightarrow \ln \left(g_{K}+d\right)_{a}$ & $\ln \left(v_{a}\right) \rightarrow v\left(i_{a}\right)$ & $\ln \left(i_{a}\right) \leftarrow \ln \left(g_{K}+d\right)_{a}$ \\
\hline EUA & 1963-03 & $\ln \left(v_{a}\right) \rightarrow \ln \left(g_{k}+d\right)_{a}$ & $\ln \left(v_{a}\right) \rightarrow \ln \left(i_{a}\right)$ & - \\
\hline Finlândia & 1963-04 & $\ln \left(v_{a}\right) \rightarrow \ln \left(g_{K}+d\right)_{a}$ & $\ln \left(v_{a}\right) \rightarrow \ln \left(i_{a}\right)$ & - \\
\hline Irlanda & 1963-04 & $\ln \left(v_{a}\right) \rightarrow \ln \left(g_{K}+d\right)_{a}$ & $\ln \left(v_{a}\right) \rightarrow \ln \left(i_{a}\right)$ & - \\
\hline Holanda* & 1963-04 & $\ln \left(v_{a}\right) \leftrightarrow \ln \left(g_{K}+d\right)_{a}$ & $\ln \left(v_{a}\right) \leftrightarrow \ln \left(i_{a}\right)$ & $\ln \left(i_{a}\right) \leftrightarrow \ln \left(g_{K}+d\right)_{a}$ \\
\hline Nova Zelândia & 1963-04 & $\ln \left(v_{a}\right) \leftrightarrow \ln \left(g_{K}+d\right)_{a}$ & - & - \\
\hline Canadá & 1963-04 & - & $\ln \left(v_{a}\right) \leftarrow \ln \left(i_{a}\right)$ & - \\
\hline Coreia do Sul* & 1966-04 & $\ln \left(v_{a}\right) \leftarrow \ln \left(g_{K}+d\right)_{a}$ & $\ln \left(v_{a}\right) \leftarrow \ln \left(i_{a}\right)$ & - \\
\hline Grécia & 1964-04 & - & $\ln \left(v_{a}\right) \leftarrow \ln \left(i_{a}\right)$ & $\ln \left(i_{a}\right) \leftrightarrow \ln \left(g_{K}+d\right)_{a}$ \\
\hline Suécia & 1963-03 & $\ln \left(v_{a}\right) \leftarrow \ln \left(g_{K}+d\right)_{a}$ & $\ln \left(v_{a}\right) \leftarrow \ln \left(i_{a}\right)$ & $\ln \left(i_{a}\right) \leftrightarrow \ln \left(g_{K}+d\right)_{a}$ \\
\hline Islândia & 1963-04 & - & - & $\ln \left(i_{a}\right) \leftrightarrow \ln \left(g_{K}+d\right)_{a}$ \\
\hline Luxemburgo & 1963-04 & - & - & $\ln \left(i_{a}\right) \leftrightarrow \ln \left(g_{K}+d\right)_{a}$ \\
\hline Áustria & 1963-04 & - & - & - \\
\hline França & 1963-04 & - & - & - \\
\hline Itália* & 1963-04 & - & - & - \\
\hline Japão & 1963-04 & - & - & - \\
\hline Noruega* & 1963-04 & - & - & - \\
\hline Reino Unido* & 1963-04 & - & - & - \\
\hline Suíça* & 1963-03 & - & - & - \\
\hline \multicolumn{5}{|c|}{ Fonte: Elaboração dos autores com base nas tabelas A3, A4 e A5 do Anexo. } \\
\hline \multicolumn{5}{|c|}{ Notas: Foi considerando um nível de significância estatística de 10\% ou menos. } \\
\hline \multicolumn{5}{|c|}{ * Países que apresentaram problemas no diagnóstico dos resíduos. } \\
\hline
\end{tabular}

para a taxa de lucro. Para a Coreia do Sul, houve uma causalidade unidirecional de Granger da taxa de investimento e da taxa de acumulação de capital para a taxa de lucro. ${ }^{9} \mathrm{Na}$ Grécia, observa-se uma relação de causalidade unidirecional da taxa de investimento para a taxa de lucro e uma relação de causalidade bidirecional entre a taxa de investimento e a taxa de acumulação de capital. Para a Suécia, além desses resultados, verificou-se causalidade unidirecional da taxa de acumulação para a taxa de lucro.

Também foram obtidos resultados que podem ser enquadrados nas duas concepções teóricas. Na Holanda, detectaram-se relações de causalidade bidirecional entre as três variáveis em estudo; na Nova Zelândia, há uma relação de causalidade bidirecional entre a taxa de lucro e a taxa de acumulação de capital. 
Por fim, na Islândia e em Luxemburgo, observou-se causalidade bidirecional entre a taxa de investimento e a taxa de acumulação de capital. Para os demais países, não foram encontradas evidências de causalidade de Granger entre as variáveis analisadas.

Uma interpretação inicial dos resultados pode ser buscada na dicotomia entre economias que são profit led e wage led (Bhaduri e Marglin, 1990). Os países com resultados que apontam para a precedência temporal da taxa de lucro em face das demais variáveis e, portanto, com um comportamento similar ao esperado pela concepção clássico-marxiana seriam economias profit led. Nelas, o aumento da taxa de lucro provoca uma elevação na taxa de investimento e na acumulação do capital. Por outro lado, os países em que a taxa de investimento precede os movimentos da taxa de lucro e da acumulação do capital seriam economias caracterizadas por um regime do tipo wage led. Nesse caso, um aumento da demanda que decorre, por exemplo, de elevações salariais ou dos gastos do governo provocaria uma elevação da taxa de investimentos e, em um segundo momento, da taxa de lucro e da taxa de acumulação.

Por sua vez, nos países que apresentam relação de bicausalidade entre a taxa de lucro e a taxa de investimento pode ter ocorrido uma mudança de regime, como a passagem de wage led para profit led ou o oposto.

Deve-se ressaltar que, para sete países, não foram encontradas relações de causalidade de Granger. Diversas razões podem explicar tais resultados, e duas possibilidades são aqui levantadas. Primeiro, nesses países os investimentos públicos podem ter um papel-chave na determinação dos investimentos privados e na acumulação de capital, o que não é captado pelo presente modelo. Uma larga literatura discute se os investimentos públicos são complementares (crowd in) ou concorrentes (crowd out) com os investimentos privados (Ribeiro e Teixeira, 2001, e Aschauer 1989). Segundo, a extensão do modelo para uma economia aberta altera o modelo e os testes empíricos podem apresentar resultados diferentes da análise aqui apresentada. Terceiro, informações podem ser perdidas ao calcular os dados em poder de paridade de compras.

Em seu conjunto, os resultados apontam que as variáveis em estudo comportaram-se de acordo com a tradição clássico-marxiana em um maior número de países. Contudo, houve países cujas relações de causalidade fo- 
ram de acordo com a concepção pós-keynesiana. Esses resultados apontam para a importância de trabalhos empíricos, os quais são capazes de apontar, de um lado, os limites das tradições teóricas heterodoxas, e, de outro, as complementaridades entre essas tradições.

\section{CONCLUSÃO}

No presente artigo foi investigada a relação de precedência temporal, causalidade no sentido de Granger, entre a taxa de lucro, a taxa de investimento e a acumulação de capital para um conjunto de 20 países da OCDE entre 1963 e 2004. A taxa de lucro, na tradição clássico-marxiana, é o principal determinante da taxa de investimento e da acumulação de capital. O procedimento desenvolvido por Toda e Yamamoto (1995) para o teste de não causalidade de Granger foi empregado para investigar se os dados desses países são consistentes com essa concepção teórica.

A evidência empírica indica que, para os casos da Austrália, Dinamarca, EUA, Finlândia e Irlanda, há uma relação de causalidade unidirecional da taxa de lucro para a taxa de investimento e da taxa de lucro para a acumulação de capital. Esse resultado é consistente com a tradição clássico-marxiana.

Contudo, nos casos do Canadá, Coreia do Sul, Grécia e Suécia, verifica-se uma relação de causalidade unidirecional da taxa de investimento para a taxa de lucro, resultado consistente com a tradição pós-keynesiana.

Por sua vez, para a Holanda, há relações de causalidade de Granger bidirecional entre as três variáveis em estudo e, para a Nova Zelândia, entre a taxa de lucro e a acumulação de capital. Os resultados, para esses dois países, são parcialmente consistentes com a tradição clássico-marxiana. Para sete países não foram obtidos resultados de causalidade de Granger nos níveis de significância estatísticos tradicionalmente empregados.

Uma possível explicação para esses resultados é que países em que movimentos da taxa de lucro precedem a mudanças na taxa de investimento e na acumulação de capital seriam profit led, enquanto os que alterações na taxa de investimento precedem a mudanças na taxa de lucro e na taxa acumulação são wage led.

Uma extensão do presente trabalho, com a consideração de economia aberta e governo, poderia contribuir para um melhor entendimento das re- 
lações existentes entre as variáveis em análise. O mesmo é verdadeiro no que tange ao emprego de outros testes empíricos, em particular de funções de impulso-resposta. Contudo, o artigo representa um passo importante para o entendimento das relações entre as variáveis em análise. O número reduzido de trabalhos empíricos é um dos principais problemas do pensamento econômico heterodoxo. A pesquisa empírica é fundamental para possibilitar o debate e o avanço dessas tradições.

\section{ANEXO}

Tabela A1: Propriedades das séries temporais, integração e cointegração

\begin{tabular}{|c|c|c|c|c|}
\hline \multirow[b]{2}{*}{ País } & \multicolumn{3}{|c|}{ Ordem de integração+ } & \multirow[b]{2}{*}{ Cointegração++ } \\
\hline & $\ln (g k+d)_{a}$ & $\ln \left(i_{a}\right)$ & $\ln \left(v_{a}\right)$ & \\
\hline Austrália & 1 & 1 & 1 & não \\
\hline Áustria & 1 & 0 & 0 & - \\
\hline Canadá & 0 & 0 & 1 & - \\
\hline Coreia do Sul & 1 & 1 & 0 & - \\
\hline Dinamarca & 1 & 1 & 0 & - \\
\hline EUA & 0 & 1 & 1 & - \\
\hline Finlândia & 0 & 0 & 1 & - \\
\hline França & 1 & 1 & 0 & - \\
\hline Grécia & 1 & 1 & 0 & - \\
\hline Holanda & 1 & 1 & 1 & $\operatorname{sim}$ \\
\hline Irlanda & 1 & 1 & 1 & não \\
\hline Islândia & 0 & 1 & 0 & - \\
\hline Itália & 0 & 1 & 1 & - \\
\hline Japão & 1 & 1 & 1 & $\operatorname{sim}$ \\
\hline Luxemburgo & 0 & 0 & 1 & - \\
\hline Noruega & 1 & 1 & 1 & não \\
\hline Nova Zelândia & 1 & 1 & 1 & não \\
\hline Reino Unido & 1 & 1 & 1 & não \\
\hline Suécia & 0 & 1 & 1 & - \\
\hline Suíça & 1 & 0 & 1 & - \\
\hline \multicolumn{5}{|c|}{ Fonte: Testes realizados pelos autores, utilizando o software econométrico EViews 5.0. } \\
\hline \multicolumn{5}{|c|}{$\begin{array}{l}\text { Notas: + Resultados baseados no teste Dickey-Fuller aumentado (ADF), considerando-se o nível de significância estatística de } 5 \% \text {. } \\
\text { Os testes de raiz unitária foram conduzidos, primeiramente, com intercepto e, depois, com intercepto e tendência linear. }\end{array}$} \\
\hline
\end{tabular}


Tabela A2: Seleção da ordem de defasagens dos modelos VAR

\begin{tabular}{|c|c|c|c|}
\hline País & $\begin{array}{l}\text { Número ótimo de defasagens } \\
\qquad(z)^{+}\end{array}$ & $\begin{array}{l}\text { Ordem de integração } \\
\text { máxima }\left(e_{m a x}\right)^{++}\end{array}$ & Modelo selecionado \\
\hline Austrália & 1 & 1 & $\operatorname{VAR}(2)$ \\
\hline Áustria & 1 & 1 & $\operatorname{VAR}(2)$ \\
\hline Canadá & 1 & 1 & $\operatorname{VAR}(2)$ \\
\hline Coreia do Sul & 1 & 1 & $\operatorname{VAR}(2)$ \\
\hline Dinamarca & 1 & 1 & $\operatorname{VAR}(2)$ \\
\hline EUA & 1 & 1 & $\operatorname{VAR}(2)$ \\
\hline Finlândia & 1 & 1 & $\operatorname{VAR}(2)$ \\
\hline França & 1 & 1 & $\operatorname{VAR}(2)$ \\
\hline Grécia & 1 & 1 & $\operatorname{VAR}(2)$ \\
\hline Holanda & 1 & 1 & $\operatorname{VAR}(2)$ \\
\hline Irlanda & 1 & 1 & $\operatorname{VAR}(2)$ \\
\hline Islândia & 1 & 1 & $\operatorname{VAR}(2)$ \\
\hline Itália & 1 & 1 & $\operatorname{VAR}(2)$ \\
\hline Japão & 1 & 1 & $\operatorname{VAR}(2)$ \\
\hline Luxemburgo & 1 & 1 & $\operatorname{VAR}(2)$ \\
\hline Noruega & 1 & 1 & $\operatorname{VAR}(2)$ \\
\hline Nova Zelândia & 1 & 1 & $\operatorname{VAR}(2)$ \\
\hline Reino Unido & 1 & 1 & $\operatorname{VAR}(2)$ \\
\hline Suécia & 1 & 1 & $\operatorname{VAR}(2)$ \\
\hline Suíça & 1 & 1 & $\operatorname{VAR}(2)$ \\
\hline
\end{tabular}

Fonte: Cálculos dos autores, utilizando o software econométrico EViews 5.0.

Notas: ${ }^{+} z$ determinado com base no Critério de Informação de Schwarz (SIC).

${ }^{++} e_{\max }$ determinado com base no teste Dickey-Fuller aumentado (ADF) - ver tabela A1. 
Tabela A3: Testes de não causalidade de Granger baseados nas estatísticas MWALD variável dependente: $\ln (g k+d)_{a}$

\begin{tabular}{|c|c|c|c|c|c|}
\hline \multirow[b]{2}{*}{ País } & \multirow[b]{2}{*}{ Período } & \multicolumn{2}{|c|}{$\begin{array}{c}\mathrm{H}_{0}: \ln \left(v_{a}\right) \text { não Granger-causa } \\
\ln (g k+d)_{a}\end{array}$} & \multicolumn{2}{|c|}{$\begin{array}{c}\mathrm{H}_{0}: \ln \left(i_{a}\right) \text { não Granger-causa } \\
\qquad \ln (g k+d)_{a}\end{array}$} \\
\hline & & $\operatorname{VAR}(2)$ & $\operatorname{VAR}(3)$ & $\operatorname{VAR}(2)$ & $\operatorname{VAR}(3)$ \\
\hline Austrália & 1963-04 & $3,944959 * *$ & $4,878007^{*}$ & 0,390797 & 0,814191 \\
\hline Áustria & 1963-04 & 0,150155 & 1,100669 & 1,223885 & 4,482923 \\
\hline Canadá & 1963-04 & 1,315281 & 2,477593 & 0,000008 & 0,289387 \\
\hline Coreia do Sul & 1966-04 & 0,514597 & 2,056356 & 0,031794 & 2,457397 \\
\hline Dinamarca & 1963-04 & 5,687961 ** & $8,768905^{* *}$ & 2,132354 & 2,616608 \\
\hline EUA & 1963-03 & $3,689009 *$ & 4,529391 & 1,346044 & 13,306726 \\
\hline Finlândia & 1963-04 & 4,273749 ** & 3,947185 & 1,850107 & 2,374861 \\
\hline França & 1963-04 & 0,093053 & 0,548524 & $3,069683^{*}$ & 4,279053 \\
\hline Grécia & 1964-04 & 1,051765 & 4,539683 & 3,020391 * & 6,443481 ** \\
\hline Holanda & 1963-04 & 0,483159 & $5,431488^{*}$ & 2,621297 & $5,770583^{*}$ \\
\hline Irlanda & 1963-04 & $4,142158 * *$ & 2,013532 & 2,086593 & 4,450130 \\
\hline Islândia & 1963-04 & 0,611478 & 1,509636 & $11,293833^{* * *}$ & $13,982783 * * *$ \\
\hline Itália & 1963-04 & 0,089383 & 0,285314 & 1,697467 & 1,693112 \\
\hline Japão & 1963-04 & 0,016879 & 1,808412 & $3,279238^{*}$ & 2,511716 \\
\hline Luxemburgo & 1963-04 & 0,469598 & 2,012567 & $4,154993^{* *}$ & 2,237361 \\
\hline Noruega & 1963-04 & 1,748436 & 3,494796 & 1,936313 & 2,368462 \\
\hline Nova Zelândia & 1963-04 & $4,215205^{* *}$ & $5,172432 *$ & 1,503773 & 1,339365 \\
\hline Reino Unido & 1963-04 & 0,010716 & 4,526984 & 0,507822 & 2,206524 \\
\hline Suécia & 1963-03 & 0,806650 & 2,508411 & $12,641985 * * *$ & $14,261040 * * *$ \\
\hline Suíça & 1963-03 & 2,112319 & 2,834629 & 1,019176 & 1,070622 \\
\hline
\end{tabular}

Fonte: Testes realizados pelos autores, utilizando o software econométrico EViews 5.0.

Notas: As estatísticas de teste reportadas seguem distribuição assimptótica chi-quadrado.

***, ** $\mathrm{e}$ * indicam rejeição da hipótese nula de não causalidade nos níveis de $1 \%, 5 \%$ e $10 \%$, respectivamente. 
Tabela A4: Testes de não causalidade de Granger baseados nas estatísticas MWALD variável dependente: $\ln \left(v_{a}\right)$

\begin{tabular}{|c|c|c|c|c|c|}
\hline \multirow[b]{2}{*}{ País } & \multirow[b]{2}{*}{ Período } & \multicolumn{2}{|c|}{$\begin{array}{c}\mathrm{H}_{0}: \ln (g k+d)_{a} \text { não Granger-causa } \\
\ln \left(v_{a}\right)\end{array}$} & \multicolumn{2}{|c|}{$\begin{array}{l}\text { H0: } \ln \left(i_{a}\right) \text { não Granger-causa } \\
\qquad \ln \left(v_{a}\right)\end{array}$} \\
\hline & & $\operatorname{VAR}(2)$ & $\operatorname{VAR}(3)$ & $\operatorname{VAR}(2)$ & $\operatorname{VAR}(3)$ \\
\hline Austrália & 1963-04 & 0,099413 & 1,034990 & 0,054718 & 1,617636 \\
\hline Áustria & 1963-04 & 0,123541 & 0,210169 & 0,965341 & 0,764260 \\
\hline Canadá & 1963-04 & 2,180158 & 1,393848 & $4,255929 * *$ & 3,289441 \\
\hline Coreia do Sul & 1966-04 & $8,787490 * * *$ & 6,403967 ** & $7,418785 * * *$ & $7,407836 * *$ \\
\hline Dinamarca & 1963-04 & 0,542388 & 0,379874 & 0,085963 & 0,367589 \\
\hline EUA & 1963-03 & 2,503652 & 4,345502 & 2,548004 & 3,722402 \\
\hline Finlândia & 1963-04 & 0,288830 & 1,910306 & 0,065430 & 1,870704 \\
\hline França & 1963-04 & $5,816290 * *$ & 3,763771 & $4,330397 * *$ & 3,255939 \\
\hline Grécia & 1964-04 & 2,217277 & 3,368018 & $2,911068^{*}$ & 4,088868 \\
\hline Holanda & 1963-04 & 0,270393 & $13,975910 * * *$ & 0,277169 & 10,653261 *** \\
\hline Irlanda & 1963-04 & 0,025756 & 0,213649 & 0,383130 & 1,639888 \\
\hline Islândia & 1963-04 & 0,120592 & 0,093113 & 0,691470 & 0,323102 \\
\hline Itália & 1963-04 & 0,026021 & 0,191691 & 0,351452 & 1,218104 \\
\hline Japão & 1963-04 & 1,394779 & 1,004795 & 2,259476 & 2,029397 \\
\hline Luxemburgo & 1963-04 & 0,372777 & 0,604494 & 0,313351 & 0,025847 \\
\hline Noruega & 1963-04 & 1,616564 & 0,949075 & 0,911156 & 0,403298 \\
\hline Nova Zelândia & 1963-04 & 0,852145 & $5,519274 *$ & 0,243587 & 3,879832 \\
\hline Reino Unido & 1963-04 & 0,000074 & 0,575701 & 0,032281 & 0,094200 \\
\hline Suécia & 1963-03 & 2,508974 & 7,827381 ** & 3,676760 * & $7,336598 * *$ \\
\hline Suíça & 1963-03 & 1,408342 & 3,645226 & 2,426740 & 3,969100 \\
\hline
\end{tabular}

Fonte: Testes realizados pelos autores, utilizando o software econométrico EViews 5.0.

Notas: As estatísticas de teste reportadas seguem distribuição assimptótica chi-quadrado.

$* * *, * * e$ * indicam rejeição da hipótese nula de não causalidade nos níveis de $1 \%, 5 \%$ e $10 \%$, respectivamente. 
Tabela A5: Testes de não causalidade de Granger baseados nas estatísticas MWALD variável dependente: $\ln \left(i_{a}\right)$

\begin{tabular}{|c|c|c|c|c|c|}
\hline \multirow[b]{2}{*}{ País } & \multirow[b]{2}{*}{ Período } & \multicolumn{2}{|c|}{$\begin{array}{c}\mathrm{H}_{0}: \ln \left(v_{a}\right) \text { não Granger-causa } \\
\qquad \ln \left(i_{a}\right)\end{array}$} & \multicolumn{2}{|c|}{$\begin{array}{l}\text { H0: } \ln \left(g k+d_{a}\right) \text { não Granger-causa } \\
\qquad \ln \left(i_{a}\right)\end{array}$} \\
\hline & & $\operatorname{VAR}(2)$ & $\operatorname{VAR}(3)$ & $\operatorname{VAR}(2)$ & $\operatorname{VAR}(3)$ \\
\hline Austrália & 1963-04 & $4,064523 * *$ & $5,430719^{*}$ & 1,071537 & 1,197337 \\
\hline Áustria & $1963-04$ & 0,275068 & 1,254954 & 0,771520 & 2,392287 \\
\hline Canadá & 1963-04 & 2,046334 & 2,883637 & 0,670042 & 1,488060 \\
\hline Coreia do Sul & 1966-04 & 0,666995 & 1,974843 & 0,003635 & 1,411835 \\
\hline Dinamarca & 1963-04 & 5,526431 ** & 7,583937 ** & 2,891181 * & 2,435884 \\
\hline EUA & 1963-03 & $3,136532 *$ & 4,580776 & 0,524872 & $16,668055^{* * *}$ \\
\hline Finlândia & 1963-04 & 3,997884 ** & 3,339843 & 0,922271 & 1,936798 \\
\hline França & 1963-04 & 0,136523 & 0,707683 & 1,837358 & 2,944736 \\
\hline Grécia & $1964-04$ & 0,899122 & 4,524753 & 1,938001 & $5,880175^{*}$ \\
\hline Holanda & 1963-04 & 1,175186 & 8,061856 ** & 2,503985 & $5,363126^{*}$ \\
\hline Irlanda & 1963-04 & 3,857577 ** & 1,981639 & 0,488032 & 2,261917 \\
\hline Islândia & 1963-04 & 0,405156 & 0,973241 & $5,840586 * *$ & $7,869889 * *$ \\
\hline Itália & $1963-04$ & 0,021123 & 0,640858 & 0,869394 & 1,146097 \\
\hline Japão & 1963-04 & 0,300799 & 1,159705 & $2,904214^{*}$ & 2,790872 \\
\hline Luxemburgo & 1963-04 & 1,258465 & 3,084411 & $2,890292^{*}$ & 0,985363 \\
\hline Noruega & 1963-04 & 1,809301 & 4,539190 & 1,161838 & 2,169742 \\
\hline Nova Zelândia & 1963-04 & 4,109011 ** & 4,256328 & 1,252914 & 1,804083 \\
\hline Reino Unido & 1963-04 & 0,177688 & 3,409413 & 0,600167 & 3,792244 \\
\hline Suécia & $1963-03$ & 0,793740 & 2,321520 & $11,540453^{* * *}$ & $14,206357^{* * *}$ \\
\hline Suíça & $1963-03$ & 2,084863 & 2,554480 & 0,135400 & 0,164276 \\
\hline
\end{tabular}

Fonte: Testes realizados pelos autores, utilizando o software econométrico EViews 5.0.

Notas: As estatísticas de teste reportadas seguem distribuição assimptótica chi-quadrado.

$\star * *, * * \mathrm{e} *$ indicam rejeição da hipótese nula de não causalidade nos níveis de $1 \%, 5 \%$ e $10 \%$, respectivamente. 


\section{NOTAS}

1. No presente trabalho, empregam-se os valores observados como proxies para os valores de longo prazo sob a condição de que a soma dos desvios de cada variável de seu valor de longo prazo seja igual ao valor do erro estimado, o qual deve seguir as hipóteses tradicionais. O comportamento dos resíduos certamente tem um papel importante na análise e será abordado na seção seis. Um procedimento alternativo seria construir variáveis de longo prazo filtrando-se as séries observadas com um dos instrumentos disponíveis (e.g., filtro $\mathrm{H}-\mathrm{P}$, alisamento polinomial etc.). No entanto, esse procedimento pode provocar distorções significativas nos dados (Ehlgen, 1998; Meyer e Winker, 2005). Os principais problemas associados ao uso de filtros são os seguintes: a) riscos de regressão espúria; b) superestimação da estrutura de defasagens no caso de séries integradas; e c) perda de poder dos testes.

2. De fato, os pré-testes realizados com nossa base de dados indicaram que (vide tabela A1 no Anexo): a) em 13 dos 20 países analisados, as séries possuem ordens de integração distintas e, portanto, não há possibilidade de cointegração; b) nos países em que as séries possuem a mesma ordem de integração, em apenas dois casos (Holanda e Japão) pode-se concluir que há cointegração. Consequentemente, o emprego da abordagem tradicional implicaria a perda de informação de longo prazo em praticamente toda a amostra.

3. O procedimento de Toda e Yamamoto (1995) é válido mesmo sob incerteza com relação à ordem de integração das séries e à existência de cointegração.

4. O Critério de Informação de Akaike (AIC) não foi empregado por tender a indicar a seleção de modelos sobreparametrizados (Verbeek, 2000, p. 254). Tal problema seria ainda mais grave no presente caso, tendo em vista o sobredimensionamento intencional decorrente do procedimento de Toda e Yamamoto (1995).

5. Com base em uma análise gráfica das variáveis, encontraram-se indícios de que cada série foi gerada ou por um processo de raiz unitária com drift ou por um processo estacionário com uma tendência determinística. Por essa razão, os testes de raiz unitária foram conduzidos, primeiramente, com intercepto e, depois, com intercepto e tendência linear. O nível de significância estatística de 5\% foi empregado para realizar os testes.

6. Por uma questão de espaço, são apresentadas apenas as conclusões dos referidos testes, mas os resultados completos estão disponíveis mediante solicitação aos autores.

7. Em geral, tanto a escolha de muitas como a de poucas defasagens podem causar problemas. Um número insuficiente de lags implica que algumas variáveis importantes estarão sendo omitidas do modelo, causando um viés de especificação que poderá levar a conclusões incorretas. Por outro lado, um número excessivo de lags implica o desperdício de observações e, geralmente, o aumento dos erros padrão dos coeficientes estimados, reduzindo a precisão dos resultados.

8. Para manter a parcimônia, os resultados dos testes de diagnóstico dos resíduos não são reportados no artigo. Obviamente, todos os detalhes estão disponíveis mediante solicitação aos autores.

9. Contudo, deve-se ressaltar que, no caso da Coreia do Sul, foram detectados problemas de autocorrelação residual e heterocedasticidade. 


\section{REFERÊNCIAS BIBLIOGRÁFICAS}

ASCHAUER, D. Does Public Capital Crowd Out Private Capital. Journal of Monetary Economics, n. 24, p. 171-188, 1989.

BHADURI, A; MARGLIN, S. Unemployment and the real wage: the economic basis for contesting political ideologies. Cambridge Journal of Economics, n. 14, p. 375-393, 1990.

CAPORALE G.; PITTIS N. Efficient estimation of cointegration vectors and testing for causality in vector autoregressions. Journal of Economic Surveys, n. 13, p. 1-35, 1999.

DICKEY, D.; FULLER, W. Distribution of the estimators for autoregression time series with a unit root. Journal of the American Statistical Association, n. 74, p. 427-431, 1979.

DUMÉNIL, G.; LÉVY, D. The Economics of the Profit Rate. Aldershot: Edward Elgar, 1993.

A stochastic model of technical change: an application to the U.S. economy. Metroeconomica, n. 46, p. 213-245, 1995.

DUTT, A. Growth, Distribution, and Uneven Development. Cambridge: Harvard Univ. Press, 1990.

EHLGEN, J. Distortionary effects of the optimal Hodrick-Prescott filter. Economics Letters, n. 61, p. 345-349, 1998.

FOLEY, D.; MICHL, T. Growth and Distribution. Cambridge: Harvard University Press, 1999.

GILES, J.; MIRZA, S. Some pretesting issues on testing for Granger noncausality. Econometrics Working Papers, Department of Economics, University of Victoria, n. 9914; 1999.

GONTIJO, C. The neoclassical model in a multiple-commodity world: a criticism on Marglin. Revista Brasileira de Economia, v. 52, n. 8, p. 335-56, 1998.

GORDON, D. Putting heterodox macro to the test: comparing Post-Keynesian, Marxian, and Social Structuralist macroeconometric models of the post-war US economy. In: GLICK, M. (Ed.). Competition, Technology and Money: Classical and Post-Keynesian Perspectives, Aldershot: Edward Elgar, 1994. p. 143-185.

GRANGER, C. Investigating causal relations by econometric models and cross-spectral methods. Econometrica, n. 37, p. 99-126, 1969.

HestON, A.; SUMMERS, R.; ATEN, B. Penn World Table Version 6.2. Center for International Comparisons of Production, Income and Prices at the University of Pennsylvania, 2006. Disponível em: http://pwt.econ.upenn.edu8. Acessado em: 3/3/2007.

—_. _ - BETTINA, A. Penn World Table Version 6.2. Center for International Comparisons of Production, Income and Prices at the University of Pennsylvania, 2006. Disponível em: http://pwt.econ.upenn.edu8. Acessado em: 3/3/2007.

JOHANSEN, S. Statistical analysis of cointegrating vectors. Journal of Economic Dynamics and Control, n. 12, p. 231-254, 1988.

KREGEL, J. The theory of economic growth. London: Macmillan, 1972.

LAVOIE, M.; RODRÍGUEZ, G.; SECCARECCIA, M. Similitudes and discrepancies in Post-Keynesian and Marxist theories of investment: a theoretical and empirical investigation. International Review of Applied Economics, n. 18, p. 127-149, 2004. 
MARGLIN, S. Growth, distribution and prices. Cambridge: Harvard University Press, 1986.

MEYER, M.; WINKER, P. Using HP Filtered Data for Econometric Analysis: Some Evidence from Monte Carlo Simulations. AStA Advances in Statistical Analysis, Springer, v. 89, n. 3, p. 303-320, 2005.

PHILLIPS, P.; PERRON, P. Testing for a unit root in time series regression. Biometrika, n. 75, p. 335-346, 1988.

RAMBALDI, A.; DORAN, H. Testing for Granger non-causality in cointegrated systems made easy. University of New England, Department of Econometrics. Working Paper, n. 88, ago. 1996.

RIBEIRO, M.; TEIXEIRA J. An Econometric Analysis of Private-Sector Investment in Brazil. Cepal Review, n. 74, p. 153-166, 2001.

TODA H.; YAMAMOTO, T. Statistical inference in vector autoregressions with possibly integrated processes. Journal of Econometrics, n. 66, p. 225-250, 1995.

VERBEEK, M. A Guide to Modern Econometrics. Nova York: John Wiley and Sons Ltd., 2000.

YAMADA, H.; TODA, H. Inference in Possibly Integrated Vector Autorregresive Models: Some Finite Sample Evidence. Journal of Econometrics, n. 86, p. 55-95, 1998.

ZAPATA, H.; RAMBALDI, A. Monte Carlo evidence on cointegration and causation. Oxford Bulletin of Economics and Statistics, n. 59, p. 285-298, 1997. 